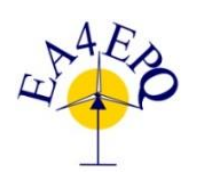

International Conference on Renewable Energies and Power Quality (ICREPQ'18)

Salamanca (Spain), $21^{\text {th }}$ to $23^{\text {th }}$ March, 2018

Renewable Energy and Pourer Quality. Fournal (RE\&PQJ)

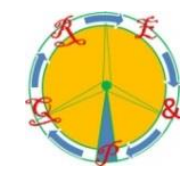

ISSN 2172-038 X, No.16 April 2018

\title{
Synchronverters used for damping inter-area oscillations in two-area power systems
}

\author{
Moshe Blau ${ }^{1}$ (deceased) and George Weiss ${ }^{2}$ \\ ${ }^{1}$ Systems Dynamics Department, Israel Electricity Company, Haifa, Israel \\ ${ }^{2}$ School of Electrical Eng., Tel Aviv Univ., Ramat Aviv 69978, Israel, gweiss@eng.tau.ac.il
}

\begin{abstract}
This research demonstrates the potential contribution of synchronverters with virtual friction to the damping of interarea oscillations and the enhancement of the transient stability of a power system. Virtual friction creates additional torque acting on the virtual rotor of the synchronverter, which is equivalent to viscous friction being present between this synchronverter and the virtual rotor of another (usually remote) synchronverter or bus bar. This function is realized by creating communication lines between synchronverters in different areas of a power system and expanding the synchronverter algorithm. Our study is based on simulations in PSS/E for an IEEE benchmark two-area network.
\end{abstract}

Key Words. synchronverter, inter-area oscillation, local mode, virtual friction, droop coefficient, power system stabilizer.

\section{Introduction}

Synchronous generators (SG) have the following useful property: once synchronized, they stay synchronized even without any control, unless strong disturbances destroy the synchronism [18]. This is one of the features that have enabled the development of the AC electricity grid at the end of the XIX-th century, and has led to the emergence of trusted control algorithms for ensuring a stable grid [13], [16], [9], [19], [11]. However, this situation is changing today due to the penetration of inverter-based power sources (mostly from renewables) into the grid [2], [4], [24], motivating many researchers to take a fresh look at grid stability using a variety of models, such as [7], [20]. The non-SG power sources threaten the stability of the power grid for various reasons, including their lack of inertia. Synchronverters have been introduced precisely to rectify this destabilizing effect of inertia-less inverters working under a variety of control algorithms. A synchronverter is an inverter controlled to emulate the behavior of a synchronous generator, see [27], [25], [26], [14]. The related concept of virtual synchronous generator was introduced earlier in [8]. Using a modest energy storage device in place of inertia, a synchronverter can emulate the inertial response typical of synchronous generators. It also includes a fast frequency drooping mechanism that can regulate its active output power in proportion to the deviation of the grid's frequency from a reference value.

The frequency droop in a synchronverter is akin to a fast acting speed governor system. It contributes to the stability in the power system by rapidly restoring the equilibrium between electromagnetic torque and mechanical torque following sudden changes in generation or load. It has been noticed ([5], [15], [27]) that synchronverters have additional benefits, that make them actually "better" than SGs: the ability to change their inertia (and other parameters) in real time, and the ability to provide practically instantneous frequency and voltage droop, while SGs and their prime movers may have huge time constants associated to their droop control loops (the inherent time constant of a modern static excitation system may be very small).

Electromechanical oscillations are an inherent phenomenon in power systems. They are usually due to small disturbances that take place frequently as a result of small variations in loads and generation [13]. Such oscillations contain multiple frequency components which are determined by generator inertia, transmission line inductance, speed governor control, generator excitation control and loads. The natural frequencies of the oscillations that are of main concern in a power system may be classified into two different modes: local and inter-area modes.

Local modes are associated with a single gene-rating plant or a small group of generators oscillating against the rest of the power system. The natural frequency of local mode oscillation is generally in the range of 1 to $2 \mathrm{~Hz}$ [16], [18]. Power system stabilizers (PSS) were originally introduced to damp this type of oscillations. A PSS adds a stabilizing damping torque to the generator's rotor by 
adding a control signal to the field voltage produced by the generator's automatic voltage regulator (AVR).

Disturbances within coherent groups of generators interconnected through weak transmission lines (i.e. high reactance) may give rise to poorly damped inter-area oscillations. The natural frequency of inter-area oscillations is typically lower than that of local modes (less than $1 \mathrm{~Hz}$ ). The damping characteristic of the inter-area mode is dictated by the tie-line strength, the nature of the loads, the power flow through the interconnection and the interaction of loads with the dynamics of generators and their associated controls. Power system stabilizers may also be designed with emphasis on damping inter-area oscillations [12]. The stability of weakly interconnected systems may be enhanced through other means, such as thyristor controlled series capacitors (TCSC) connected along the tie-line [21] or shunt connected static Var compensators (SVC) [10] among others.

Another problem of concern to power utilities is that of transient stability. This refers to the ability of synchronous machines of an interconnected power system to remain in synchronism after being subjected to a major disturbance [13]. The most severe disturbance is a solid three-phase symmetric short circuit in a transmission line followed by its inevitable disconnection by protective fault clearing de- vices. Although such faults are rare, a network must be designed and operated to withstand such events regardless of their frequency. The transient stability of a power system depends on the ability to restore the equilibrium between electromagnetic and mechanical torque of each SG after the disturbance. A fundamental factor in this problem is the manner in which the power outputs of SGs vary as their relative rotor angles change. The system response to large disturbances may involve extreme excursions of generator rotor angles. Instability may ensue as a result of increasing angular divergence of some generators leading to their loss of synchronism.

The virtual friction function in a synchronverter is designed to add a damping component to the inverter's output power in proportion to the difference between the frequencies of two separate coherent groups of generators interconnected through a relatively long tie-line. It creates additional torque acting on the virtual rotor of the synchronverter, which is equivalent to viscous friction being present between this synchronverter and the virtual rotor of another (usually remote) synchronverter. The virtual friction can also act to emulate viscous friction against the rotor of a real SG (the friction torque will act only on the synchronverter rotor). This function has a stabilizing effect on inter-area oscillations in addition to

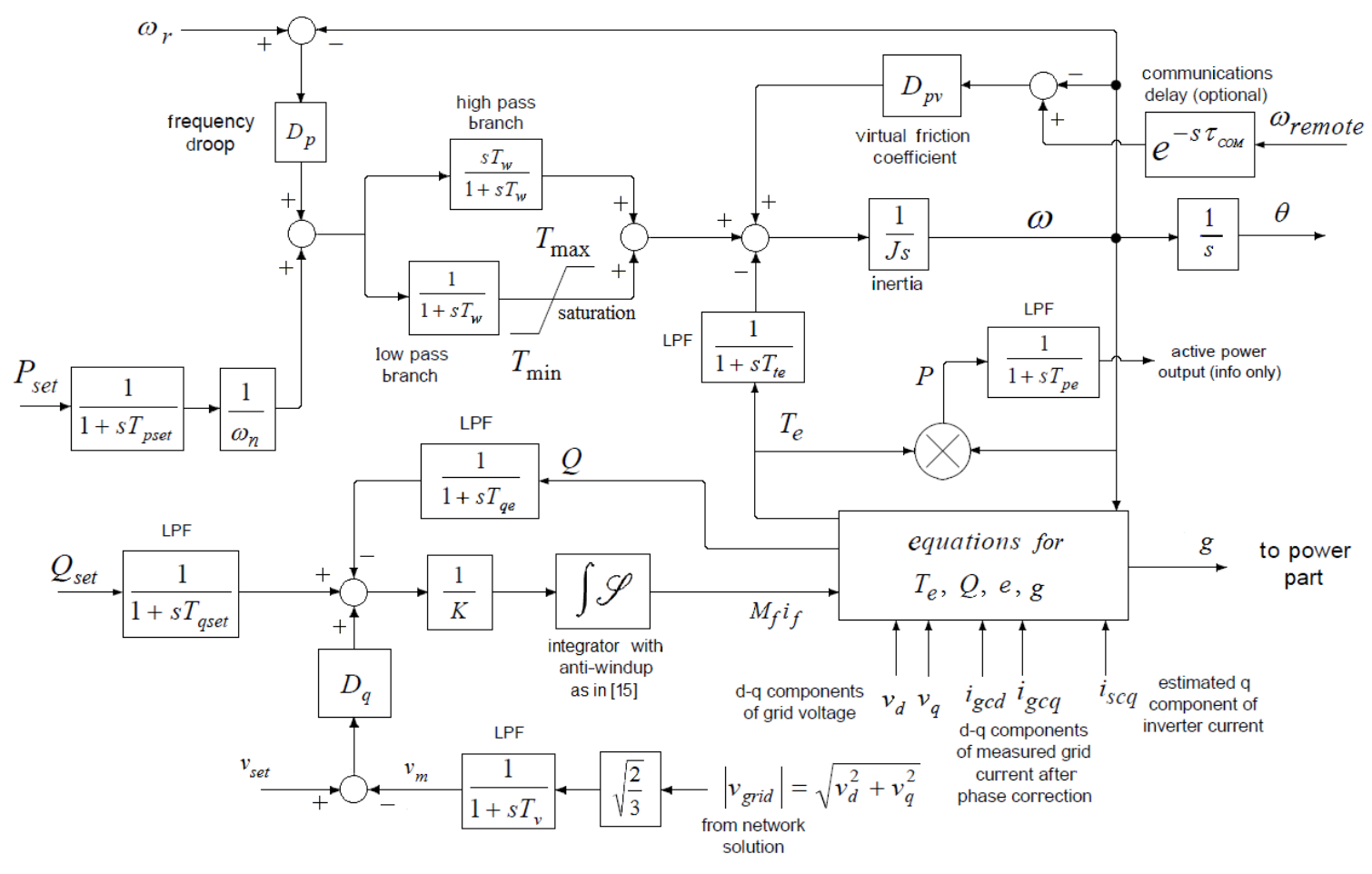

Fig. 1. Simulation model of the improved synchronverter (the electronic part) following [15], with the virtual friction also included. The virtual inductor from [15] is not shown, but it is part of the block of equations giving the average voltages $g$ (a 3 dimensional vector) produced by the switches in the 3 inverter legs. For the details about the integrator with anti-windup and the blocks near it we refer to [15]. The decomposition of the active torque into high and low-pass branches, with a saturation, is explained in [22]. 
the frequency drooping mechanism. Virtual friction was introduced in [5], [6]. A mathematical investigation for the simplest possible case (two identical generators and a load) is contained in [23]. It uses earlier results about the stability of a SG connected to an infinite bus [14]. The aim of this paper is to investigate the effects of virtual friction on some realistic grid models.

\section{The model used in the simulations}

The two-area system that was selected for this study is based on an artificial two-area four generator IEEE test benchmark [17]. This benchmark system is itself based on a model created for a research report commissioned from Ontario Hydro by the Canadian Electrical Association to study the different types of oscillations that occur in interconnected power systems [18].

After skipping many details, we show in Figure 1 our model of a synchronverter, based on [5], [15], [22], [27]. For the details of the block diagram (algorithm) and how it is motivated by the structure of a SG, we refer to [5], [15], [27]. We have not shown the blocks needed for selfsynchronization (the start-up process), see [26]. The virtual inductor and virtual capacitor in each phase (see Figure 2) are motivated and explained in [15]. The synchronous internal voltage $e_{q}$ together $e_{d}=0$ is transformed by the inverse Park transformation into $e=\left(e_{a}, e_{b}, e_{c}\right)$ and from here the algorithm computes $g=\left(g_{a}, g_{b}, g_{c}\right)$ by the simple formula $g=\frac{1}{n}((n-1) v+e)$, where $v$ is the local grid voltage, see [15]. These signals $g$ are provided as the desired average voltages to the PWM generator. OK

We say a few words about the output circuit of a synchronverter, since this is important for our simulations. The literature proposes different LC (or LCL) filter design guidelines for grid connected inverters [1], [3], [4], [20], [25]. The differences in guidelines seem to be a result of differences in the proposed inverter topologies, treatment of the neutral point, modulation methods (PWM, SPWM or
SVM) and plant configurations. We extract the following general guidelines:

1. The inverter-side inductance is determined by the maximum allowable current ripple at the output of the inverter in relation to the rated current of the inverter.

2. The filter capacitance should be selected so that the resonant frequency of the filter lies between the nominal grid frequency and the switching frequency of the inverter. It is also recommended that the capacitance be a fraction of the base capacitance at rated conditions [3].

Figure 2 shows a single phase diagram of the 3 phase inverter with virtual components in series (capacitor and inductor) connected to the utility grid through an LC filter, an LV/MV isolation transformer and an MV/HV station step-up transformer. The figure also shows the presumed PCC at the high voltage side of the station transformer. The nominal grid voltage at the output of the LC filter is 690 Volts or 398.4 Volts phase to neutral (rms).

Figure 3 is a simplified representation of a two-area power system with one synchronverter in each area. Each of the two areas in the figure has also two synchronous generators connected to an interconnecting tie-line through intermediate bus bars. Each synchronverter is represented by an inverter, a virtual inductance and the inductance of the LC filter. The virtual friction function is incorporated into both synchronverters by modifying the equations of rotational motion of the virtual rotors as follows:

$$
\begin{aligned}
& \frac{d \omega_{1}}{d t} J_{1}=T_{m 1}-T_{e 1}-D_{p 1}\left(\omega_{1}-\omega_{r}\right)-D_{p v}\left(\omega_{1}-\omega_{2}\right), \\
& \frac{d \omega_{2}}{d t} J_{2}=T_{m 2}-T_{e 2}-D_{p 2}\left(\omega_{2}-\omega_{r}\right)-D_{p v}\left(\omega_{2}-\omega_{1}\right),
\end{aligned}
$$

where $\omega_{1}$ and $\omega_{2}$ are the angular velocities of the virtual rotors of synchronverters $\mathrm{S} 1$ and $\mathrm{S} 2, D_{p 1}>0$ and $D_{p 2}>0$ are their corresponding frequency droop constants and

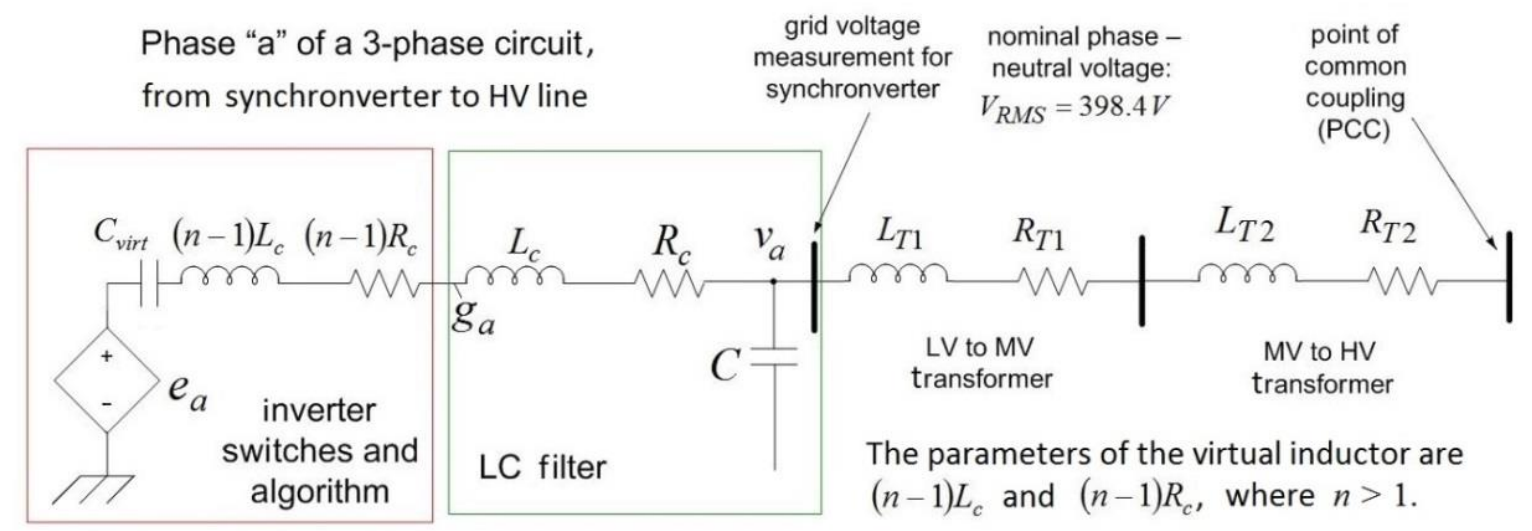

Fig. 2. Single phase diagram of a 3-phase inverter with virtual components connected to the utility grid 
$D_{p v}>0$ is the virtual friction coefficient (we took the same value in both synchronverters, but they may also differ). In our equations, $J_{k}$ are the moments of inertia, $T_{m k}$ are the virtual active torques and $T_{e k}$ are the virtual electric torques $(k=1$ or 2$)$.

\section{Simulation results}

We performed a series of time domain simulations to demonstrate the potential impact that synchronverters with virtual friction could have on the mitigation of inter-area oscillations and the enhancement of transient stability in a two-area power system. The simulations were performed using the Siemens-PTI PSS/E (power systems simulator for engineers) software package. Since its introduction in 1976, PSS/E has become one of the most widely used commercial programs for the study of power systems dynamics. For this study a dynamic simulation model of the synchronverter was integrated into the PSS/E software as a synchronous generator User Model. To obtain our first test system, the 4 generator system in the IEEE benchmark [17] was modified to accommodate two additional SGs, becoming a two-area 6 generator system. In the second test system, two of the generators (marked as G5 and G6 in Figure 3) have been replaced with two identical synchronverters (marked as S5 and S6 in Figure 3) of the same MVA rating with a nominal power factor of 0.9 and step-up transformers with approximately the same equivalent short circuit impedance, which in the case under consideration has a value of 0.17 per unit. The virtual friction function was based on the frequency measured at bus bars 6 and 10 .

For lack of space, we report here on only one simulation experiment. The transient stability of both systems was tested by simulating a 100 millisecond $(6$ cycles) solid three-phase fault in circuit number \#1 (CKT1) of the tieline next to load bus 7. After the 6 cycle period the fault was cleared by tripping the shorted circuit CKT1. This fault represents the worst possible case involving a single tie-line circuit. The simulations were repeated for two different tie-line stress levels. The first set of simulations was performed for a relatively low initial power flow of 200 MW from bus 7 to bus 9 while in the second set the initial power flow was $300 \mathrm{MW}$.

The virtual friction coefficient was adjusted to yield effective damping of inter-area oscillations, without risking the overload of the synchronverters during the most severe disturbance under consideration. The constants related to the voltage and reactive power control loop $\left(K\right.$ and $\left.D_{q}\right)$ were adjusted experimentally to allow for rapid terminal voltage recovery after the clearing of a severe fault while preventing an excessive voltage overshoot. The frequency droop constant $D_{p}$ corresponds to a speed regulation coefficient of $3 \%$. The minimum and maximum values of the virtual magnetic flux linkage were set to yield a steadystate back EMF $e_{q}$ between $30 \%$ and $200 \%$ of its value at the rated MVA of the synchronverter at a power factor of 0.9. The remaining parameters were set according to [5].

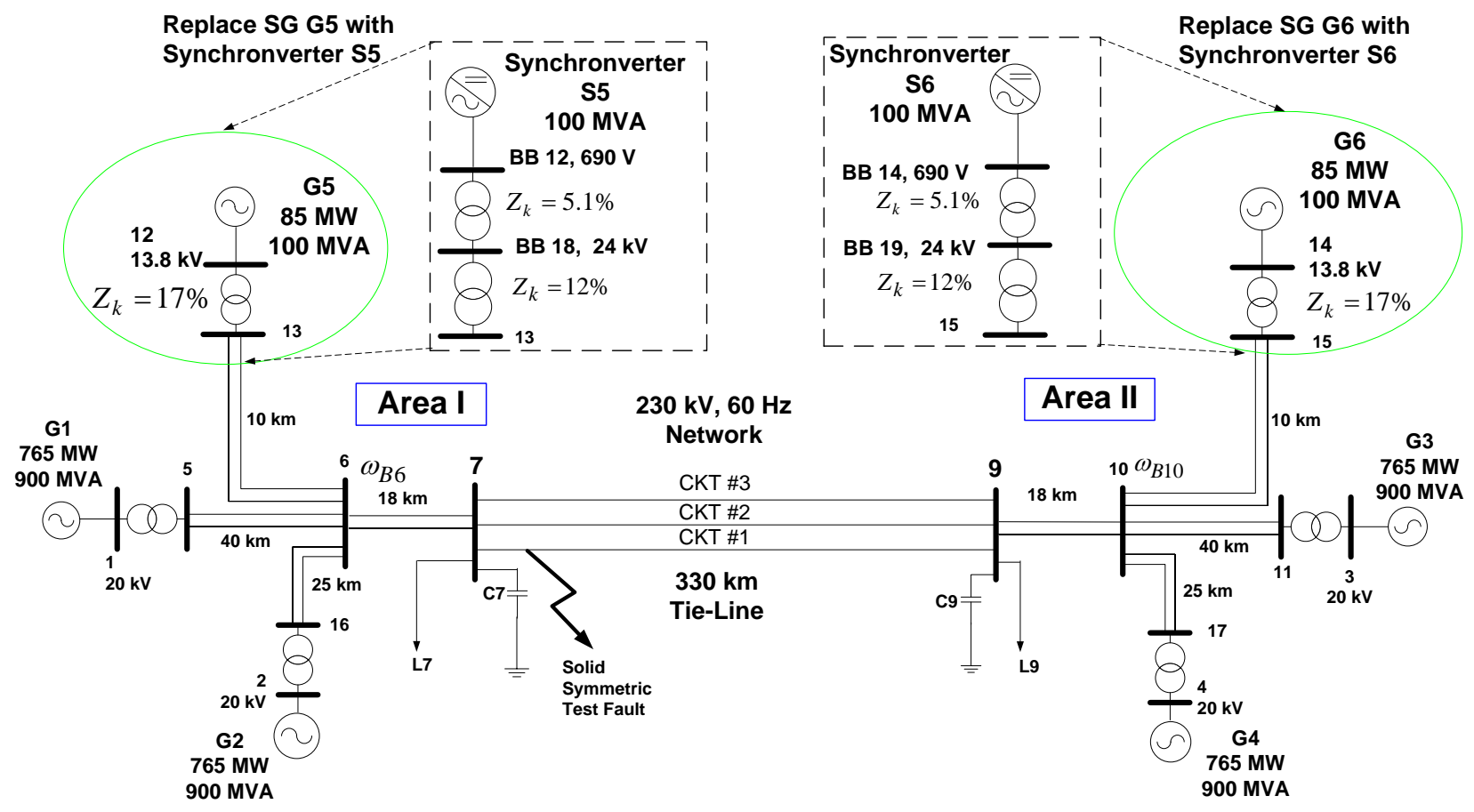

Fig. 3. Our two-area system containing either 3 SGs or 2 SGs and one synchronverter in each area 
The excitation system selected for the SGs was a DC rotating type exciter. This type of exciter and its associated AVR are characterized by a relatively slow time response to changes in SG terminal voltage. This may improve small signal stability but it is detrimental to the transient and the voltage stability of the interconnected system [13]. The power system stabilizer model used in conjunction with the exciter AVRs was a general purpose speed sensitive PSS.

Figure 4 (green and blue curves) shows the active power flow from area I to area II when the initial power flow through the interconnection is $300 \mathrm{MW}$, when the system is composed of $6 \mathrm{SG}$ without and with PSS. We see that the system becomes unstable with loss of synchronism approximately 1.8 seconds after the fault is cleared. By contrast, if two synchronverters with virtual friction are present (red and blue curves), then the system remains stable, with and without PSSs. Moreover, the oscillations in power flow are suppressed within 5 seconds. If the initial power flow is reduced to $200 \mathrm{MW}$, then the fault would cause severe tie-line power oscillations for several seconds, without loss of synchronism (not shown here for lack of space).

\section{Conclusions}

By simulating short circuits lasting for $0.1 \mathrm{sec}$ in a twoarea power system including conventional generators and one synchronverter in each area, we have observed that the system recovery is much quicker and smoother when the virtual friction feature is present. We have tried this with or without PSS in the generators, the results are similar.

\section{References}

[1] K.H. Ahmed, S.J. Finney and B.W. Williams, "Passive filter design for three-phase inverter inter-facing in distributed generation", IEEE Conf. on Compatibility in Power Electronics (CPE2007), Gdansk, Poland, May 2007.

[2] J. Alipoor, Y. Miura and T. Ise, "Distributed generation grid integration using virtual synchronous generator with adaptive virtual inertia", IEEE Energy Conversion Congress and Exposition (ECCE13), Denver, CO, pp. 4546-4552, Sept. 2013.

[3] R.M. Beres, X. Wang, M. Liserre, F. Blaabjerg and C.L. Bak, "Review of Passive Power Filters for ThreePhase Grid-Connected Voltage-Source Converters", IEEE J. of Emerging and Selected Topics in Power Electronics, vol. 4, March 2016.

[4] F. Blaabjerg, R. Teodorescu, M. Liserre and A.V. Timbus, "Overview of control and grid synchronization for distributed power generation systems", IEEE Trans. on Industrial Electronics, vol. 53, 2006, pp. 1398-1409.

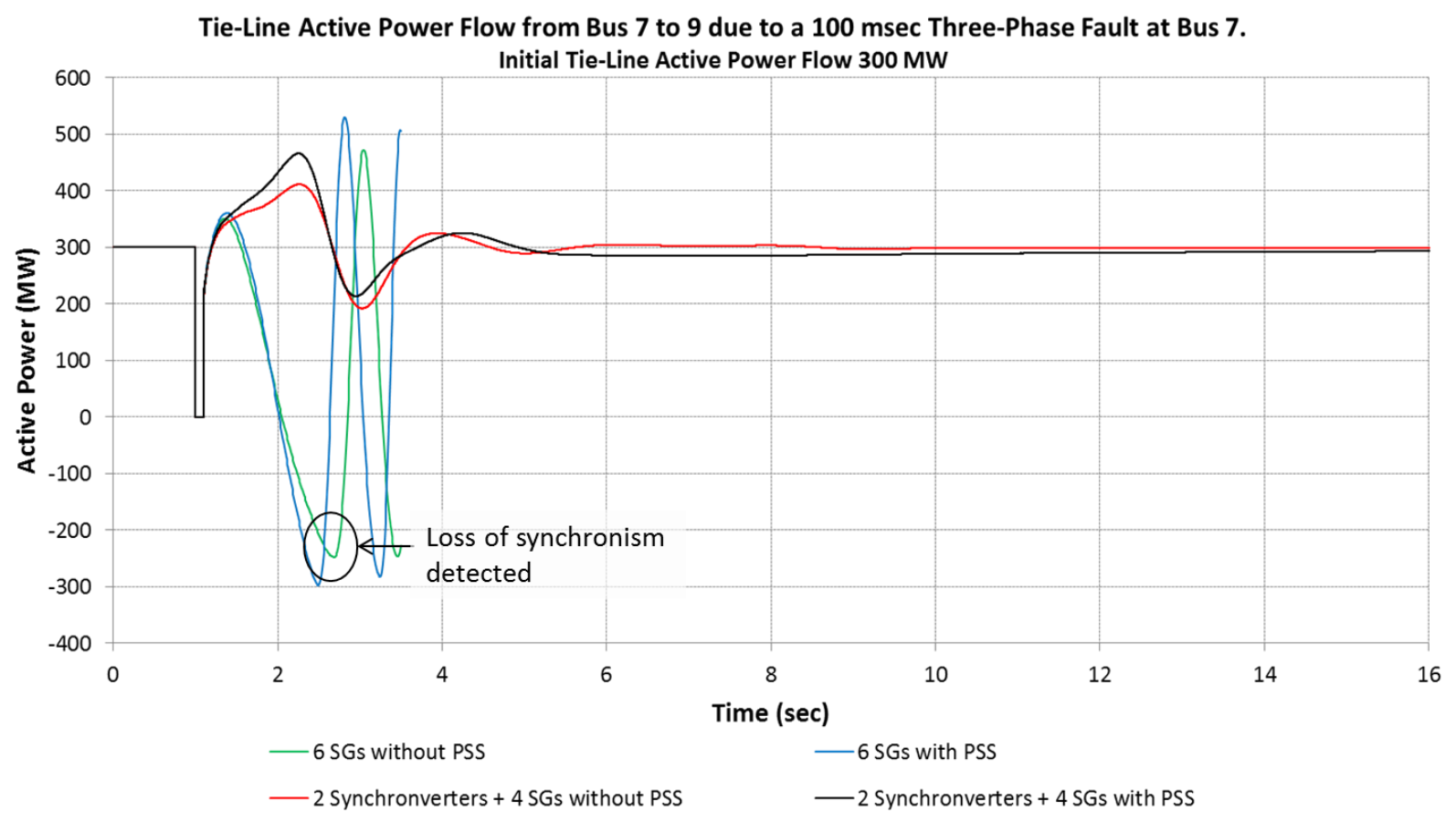

Fig. 4. Tie-line active power flow from area I to area II due to a three-phase fault (short circuit) in circuit \#1 of the interconnecting tie-line CKT1 from Figure 3, occurring at $\mathrm{t}=1 \mathrm{sec}$, and lasting for $0.1 \mathrm{sec}$ 
[5] E. Brown, "A study of the use of synchronverters for grid stabilization using simulations in SimPower", MSc Thesis, Tel Aviv University, August 2015.

[6] E. Brown and G. Weiss, "Using synchronverters for power grid stabilization", IEEE 28-th Conv. of Electr. and Electronics Engineers in Israel, Eilat, Israel, Nov. 2014.

[7] F. Dörfler and F. Bullo, "Synchronization and transient stability in power networks and nonuniform Kuramoto oscillators", SIAM J. Control and Optim., vol. 50, 2012, pp. 1616-1642.

[8] J. Driesen and K. Visscher, "Virtual synchronous generators", IEEE Power and Energy Society General Meeting - Conversion and Delivery of Electrical Energy, Pittsburg, PA, 2008, pp. 1-3, 2008.

[9] O.I. Elgerd, "Electric Energy Systems Theory, An Introduction", McGraw-Hill, 1982.

[10] L. Gerin-Lajoie, G. Scott, S. Breault, E. V. Larsen, D. H. Baker and A. F. Imece, "Hydro-Quebec Multiple SVC Application Control Stability Study", IEEE Transactions on Power Delivery, vol. 5, 1990, pp. 1543-1551.

[11] J.J. Grainger and W.D. Stevenson, "Power System Analysis", McGraw-Hill, New York, 1994.

[12] M. Klein, G.J. Rogers. S. Moorty and P. Kundur, "Analytical Investigation of Factors Influencing Power System Stabilizers Performance", IEEE Trans. on Energy Conversion, vol. 7, 1992, pp. 382-390.

[13] P. Kundur, "Power System Stability and Control", McGraw-Hill, Palo Alto, California, 1994.

[14] V. Natarajan and G. Weiss, Almost global asymptotic stability of a grid-connected synchronous generator, available on ResearchGate, and on arXiv.

[15] V. Natarajan and G. Weiss, Synchronverters with better stability due to virtual inductors, virtual capacitors and anti-windup, IEEE Trans. Industrial Electronics, vol. 64, 2017, pp. $5994-6004$.

[16] B. Pal and B. Chaudhuri, "Robust Control in Power Systems", Power Electronics and Power Systems Series, Springer-Verlag, 2005.
[17] R.A. Ramos and R. Kuiava, "IEEE PES Task Force on Benchmark Systems for Stability Controls Report on the 2-area, 4-generator system", Version 5 - June 7th, 2014, available from http://resourcecenter.ieeepes.org/pes/-product/technical-reports/

[18] G. Rogers, "Power System Oscillations", Springer Science + Business Media, New York, 2000.

[19] P.W. Sauer and M.A. Pai, "Power System Dynamics and Stability". Stipes Publishing, Champaign, IL, 1997.

[20] J. Schiffer, R. Ortega, A. Astolfi, J. Raisch and T. Sezi, "Conditions for stability of droop-controlled inverterbased microgrids", Automatica, vol. 50, 2014, pp. 24572469.

[21] S. Warathe and R.N. Patel, "Power Oscillation Compensation by TCSC", International Conf. on Advanced Computing and Communication Systems (ICACCS -2015), Coimbatore, India, Jan. 2015.

[22] G. Weiss and V. Natarajan, "Modifications to the synchronverter algorithm to improve its stability and performance", Proc. Int. Symp. Power Electronics (Ee2017), Novi Sad, Serbia, Oct. 2017.

[23] G. Weiss and E. Venezian, "Stability analysis for coupled synchronous generators with virtual friction", Proc. of DSP 2017, London, August 2017.

[24] M. Yu, A. Dysko, C. Booth, A. Roscoe, J. Zhu and H. Urdal, "Investigations of the constraints relating to penetration of nonsynchronous generation in future power systems," Protection, Automation and Control World Conf., Glasgow, UK, July 2015.

[25] Q.-C. Zhong and T. Hornik, "Control of Power Inverters in Renewable Energy and Smart Grid Integration", John Wiley \& Sons, Ltd, West Sussex, United Kingdom, 2013.

[26] Q.-C. Zhong, P.-L. Nguyen, Z. Ma and W. Sheng, "Self-synchronized synchronverters: Inverters without a dedicated synchronization units", IEEE Trans. Power Electronics, vol. 29, 2014, pp. 617-630.

[27] Q.-C. Zhong and G. Weiss, "Synchronverters: Inverters that mimic synchronous generators", IEEE Trans. on Industrial Electronics, vol. 58, 2011, pp. 1259-1267. 